\title{
Measurements of Multi-boson production, Trilinear and Quartic Gauge Couplings with the ATLAS detector
}

\author{
Maurice Becker ${ }^{1, a}$, on behalf of the ATLAS Collaboration \\ ${ }^{1}$ Institute of Physics, Johannes Gutenberg University Mainz
}

\begin{abstract}
The ATLAS collaboration has carried a set of measurements that provide stringent tests of the electroweak sector of Standard Model, specifically on di- and multiboson production cross sections and on triple and quartic gauge-boson couplings. Such measurements include cross sections for $\mathrm{WV}(\mathrm{V}=\mathrm{W}$ or $\mathrm{Z})$ production in the leptonic or semileptonic channels, the production of a $\mathrm{W}$ or $\mathrm{Z}$ boson in association with photons, a $\mathrm{Z}$ boson in the vector-boson fusion channel and two same-charge $\mathrm{W}$ bosons in the vectorboson scattering channel. These measurements are compared to (N)NLO predictions of the Standard Model and provide model-independent constraints on new physics, by setting limits on anomalous gauge-boson couplings.

An analysis of the inclusive four-lepton lineshape has been carried out in the mass range from 80 to $1000 \mathrm{GeV}$, where several distinct physics processes give rise to the production of 4-lepton final state, namely the single $\mathrm{Z}$ resonant processes, the Higgs production at $125 \mathrm{GeV}$, as well as continuum $\mathrm{ZZ}$ production processes with $q \bar{q}$ and gg initial states.
\end{abstract}

\section{Introduction}

Electroweak (EW) precision measurements at LHC provide a good way to test the expectations of the Standard Model in the EW sector. Furthermore many diboson processes play an important background role for other analyses like measurements of Higgs boson properties or searches for signatures beyond the Standard Model. Comparing expectations of EW processes with measurements allows indirect searches for new physics. Deviations from SM gauge interactions, like anomalous triple gauge couplings (aTGCs), could lead to changes in the production cross section, especially in the high boson pair mass range. One can not only search for anomalous couplings, but also the expected triple and quartic gauge coupling can be studied at proton proton collisions at the LHC like Vector Boson Fusion (VBF) or Vector Boson Scattering (VBS). Especially measuring the VBS cross section is a good way to probe EW symmetry breaking. This note presents the latest results of diboson cross section measurements using data from the ATLAS detector [1] at the LHC from 2011 and 2012 with $\sqrt{s}=7 \mathrm{TeV}$ and $8 \mathrm{TeV}$ with an integrated luminosity of $4.7 \mathrm{fb}^{-1}$ and $20.3 \mathrm{fb}^{-1}$ respectively. Also VBF and VBS measurements are presented together with the first evidence of quartic gauge coupling obtained at LHC.

\footnotetext{
ae-mail: maurice.becker@cern.ch
} 


\section{Experimental Setup and Physics Object Definitions}

Every analysis that is presented here measures the cross section of processes with at least one lepton in addition with other objects like jets, photons or missing transverse momentum $\left(E_{\mathrm{T}}^{\text {miss }}\right)$. How these objects are measured within the ATLAS detector shall be shortly described.

Electrons are build from tracks in the inner detector that are matched to clusters in the electromagnetic calorimeter, while muons are reconstructed by matching the inner detector tracks with the tracks of the muon spectrometer. The typical kinematic range where the leptons are measured is above a $p_{\mathrm{T}}$ of $15 \mathrm{GeV}$ in a absolute pseudorapidity ${ }^{1}$ region smaller than 2.47 , that is covered by the tracking system. Due to the confinement of quarks in the Standard Model quarks can not exist isolated. As a result quarks hadronize to collimated bundles of particles (jets). Jets are build by using special cluster algorithms e.g. the anti- $k_{t}$ algorithm [2].

Due to the fact that neutrinos do not leave signatures in the detector it is possible to measure the size of missing transverse momentum using momentum conservation.

\section{Diboson production cross section measurements}

\section{1 $\mathbf{W} \gamma$ and $Z \gamma$ cross section measurement}

This analysis measured the integrated and differential fiducial cross sections for $\mathrm{W}$ (or Z) boson production in association with a high-energy photon [3], looking at leptonically decaying $\mathrm{W}$ or $\mathrm{Z}$ bosons in ATLAS data recorded 2011 at $\sqrt{s}=7 \mathrm{TeV}$ with $4.6 \mathrm{fb}^{-1}$. In addition to production of a $\mathrm{W}$ and $\mathrm{Z}$ boson via the s, u- and t-channels, the signal receives also contributions from $\mathrm{W}$ boson plus quark (gluon) when the quark (gluon) fragments to a photon.

The selection of $\mathrm{W} \gamma$ events is done by requiring exactly one high $p_{\mathrm{T}}$ lepton, one high $p_{\mathrm{T}}$ photon, high $E_{\mathrm{T}}^{\text {miss }}$ and high transverse mass of the lepton- $E_{\mathrm{T}}^{\text {miss }}$ system. To select $\mathrm{Z} \gamma$ events two high $p_{\mathrm{T}}$ leptons from the same flavor but opposite charge are required that form an invariant mass near the $\mathrm{Z}$ boson mass, accompanied by a high energetic photon. In order to subtract background from events where the photon is radiated from a lepton in a bremsstrahlung process, it is required that the photon is clearly separated from the lepton. $\mathrm{Z} \gamma$ events where the $\mathrm{Z}$ boson decays to neutrinos are selected by only requiring an high $E_{\mathrm{T}}^{\text {miss }}$ and an isolated photon.

The main background contributions are given by $\mathrm{W}+$ jets events where the photon results from a $\pi^{0}$ decay, $\mathrm{Z}$ events that mimic a $\mathrm{W} \gamma$ signal and $\gamma+$ jets events where a jet fakes a lepton and $E_{\mathrm{T}}^{\mathrm{miss}}$ comes from e.g. mis-measured jet energies. These backgrounds are estimated using a two-dimensional sideband method where the contribution is measured in orthogonal control regions and extrapolated to the signal region.

The results of the differential cross-section are shown in Figure 1 as a function of the $E_{T}^{\gamma}$. The distributions are shown at particle level where detector effects are corrected for (unfolded). The cross section is given in an inclusive region, including all additional jets that might occur in an event and in an exclusive region that adds an extra requirement that there are no additional jets in the event since the exclusive region is more sensitive to anomalous triple gauge couplings. The measured cross section is compared to different theory predictions from SHERPA [4], ALPGEN [5] and MCFM [6]. It can be seen that MCFM leads to worse results in higher $E_{T}^{\gamma}$ regions compared to SHERPA and ALPGEN for the $\mathrm{W} \gamma$ case due to the fact that this region is more sensitive to higher jet multiplicities that are not included in MCFM. Besides of this a good agreement between data and theory prediction can be seen and therefore also limits on anomalous triple gauge couplings were set.

\footnotetext{
${ }^{1}$ ATLAS uses a right-handed coordinate system where the $\mathrm{z}$-axis points along the beam axis, the $\mathrm{x}$-axis to the center of the LHC and the y-axis upwards. In the transverse plain cylindrical coordinates are used and the pseudorapidity is defined as $\eta=-\ln [\tan (\theta / 2)]$, where $\theta$ is the polar angle.
} 


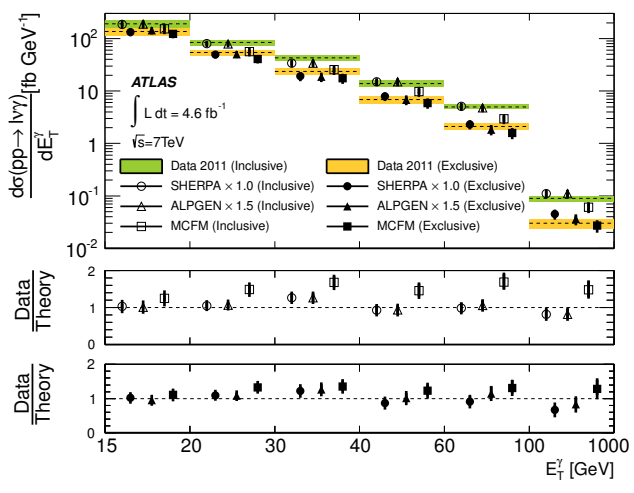

(a)

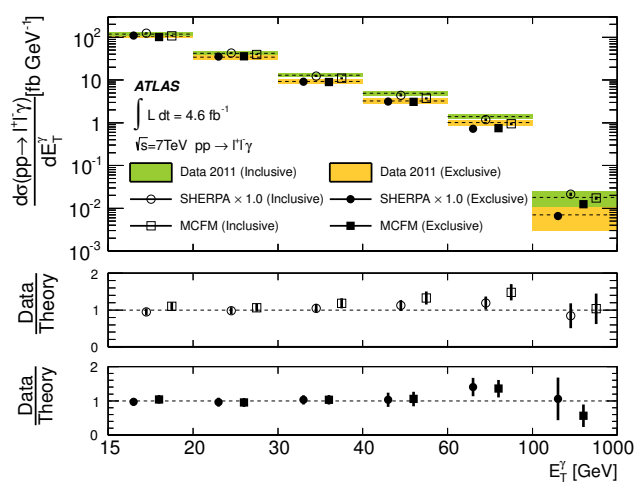

(b)

Figure 1. Differential cross section of (a) $p p \rightarrow W \gamma \rightarrow l v \gamma$ and (b) $p p \rightarrow Z \gamma \rightarrow l^{+} l^{-} \gamma$ processes, using combined electron and muon measurements in an exclusive $\left(N_{\text {jet }}=0\right)$ and inclusive $\left(N_{\text {jet }} \geq 0\right)$ region [3]. Included is besides the measured result using data theory predictions using MCFM, SHERPA and ALPGEN are shown.

\subsection{Measurement of $W^{ \pm} Z$ production decaying leptonically}

A further process of the electroweak interaction is the production of a $\mathrm{W}$ and a $\mathrm{Z}$ boson where both decay leptonically to electron or muons. In this analysis [7] events in $13 \mathrm{fb}^{-1}$ data from 2012 at $\sqrt{s}=8 \mathrm{TeV}$, are selected with two same flavor, opposite charge leptons within the $\mathrm{Z}$ mass window $\left(\left|m_{l l}-91.1876\right|<10 \mathrm{GeV}\right)$ and one additional lepton that forms together with the missing transverse momentum a W boson. One background that passes the selection criteria comes from events with three real electrons, amounting to $8 \%$ of the selected events (e.g. ZZ events where one lepton escapes the detector acceptance and thus faking $E_{\mathrm{T}}^{\mathrm{miss}}$ which is estimated via Monte Carlo simulation). Another background comes from processes where one lepton is faked (e.g. jet faking an electron) which is estimated using a data driven method and amounts about $17 \%$ of the selected events.

The integrated cross section, measured to $20.3_{-0.7}^{+0.8}(\text { stat. })_{-1.1}^{+1.2}(\text { syst. })_{-0.6}^{+0.7}($ lumi. $) \mathrm{pb}$, is in good agreement with the theory prediction of MCFM of $20.3 \pm 0.8 \mathrm{pb}$.

\subsection{Measurement of $W^{+} W^{-}$production decaying leptonically}

The $W^{+} W^{-} \rightarrow l^{+} v l^{-} v$ cross section is measured by selecting events with two leptons with opposite charge and large missing transverse momentum [8]. Since top pair production $(t \bar{t})$ would lead to the same signature it is required that there is no jet with $p_{\mathrm{T}}>25 \mathrm{GeV}$ in an event. With this cut a large fraction of the $t \bar{t}$ background is rejected as it is shown in Figure 2(a). To reject Z+jets events, where a miscalibrated jet could fake $E_{\mathrm{T}}^{\text {miss }}$ and the charge of the leptons is misidentified, it is required that the dilepton invariant mass is outside of the $\mathrm{Z}$ mass window. The remaining $t \bar{t}$ and $\mathrm{Z}+$ jets background is estimated using data driven techniques. The measured total cross section is $71.4_{-1.2}^{+1.2}(\text { stat. })_{-4.4}^{+5.0}(\text { syst. })_{-2.1}^{+2.2}($ lumi. $)$ pb and shown together with a theory prediction in Figure 2(b). It can be seen that the combined cross section differs by about $2.1 \sigma$ from the SM prediction. Possible explanations for this difference could be missing NNLO corrections in QCD [9] and/or jet-veto resummation [10]. 


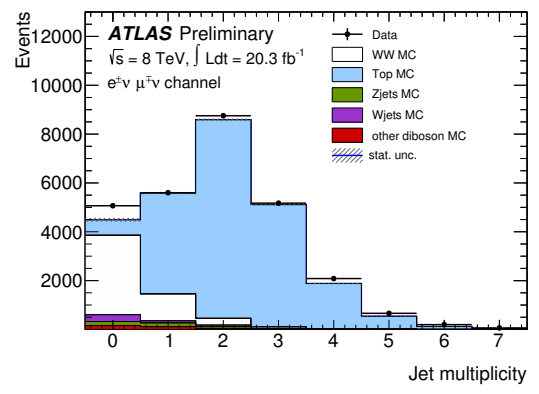

(a)

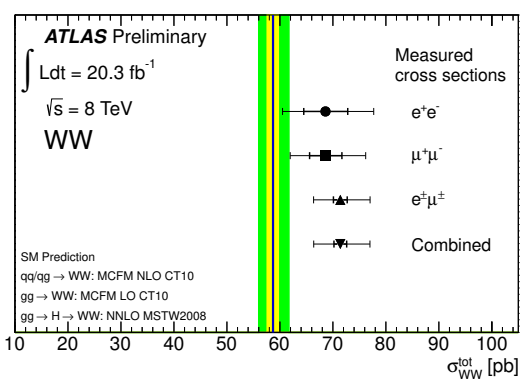

(b)

Figure 2. (a): Jet multiplicity for $W^{+} W^{-}$events in the $e^{ \pm} v \mu^{\mp} v$ channel without applying a jet veto [8]. (b): Measured and predicted $W^{+} W^{-}$cross section for three different channels together with their combination [8]. The green and yellow bands represent the PDF and the total theory uncertainties.

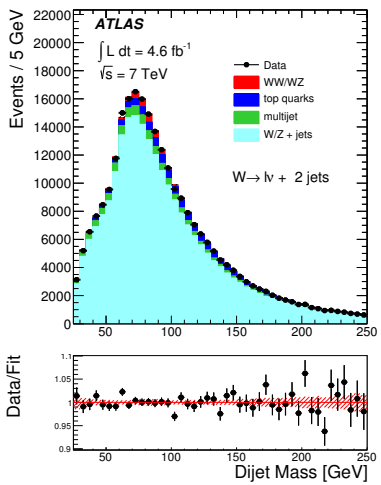

(a)

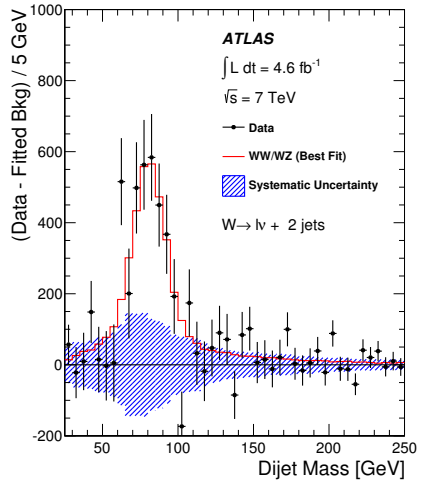

(b)

Figure 3. (a): Distributions of the dijet invariant mass for the sum of the electron and muon channels after the likelihood fit in semileptonic decaying WZ events. (b): Distribution of the background-subtracted data for the sum of the electron and muon channels in semileptonic decaying WZ events [11].

\subsection{Measurement of $W W / W Z$ production decaying semileptonic}

An analysis of the WZ/WZ production where one of the bosons decays hadronically has also been performed using the 2011 data of ATLAS [11]. The selection requires high $E_{\mathrm{T}}^{\text {miss }}$, a high $p_{\mathrm{T}}$ lepton forming together high transverse mass. Events are selected where there is one leptonically decaying $\mathrm{W}$ boson and hadronically decaying $\mathrm{Z}$ or $\mathrm{W}$ boson. Due to the jet energy resolution it is not possible to distinguish between the bosons. It is also not possible to distinguish this signature from background where there is only one $\mathrm{W}$ or $\mathrm{Z}$ boson with additional jets from higher order QCD processes as it can be seen in Figure 3(a). In order to extract the signal, the background is fitted with a likelihood approach where the shape is taken from simulation. The result after subtracting the background can be seen in Figure 3(b). The measured cross section is $68 \pm 7$ (stat.) \pm 19 (syst.) pb and in good agreement with the theory expectation of $61.1 \pm 2.2 \mathrm{pb}$ from MC@NLO [12]. The large systematic uncertainty on the measured result is dominated by the uncertainties of the jet energy scale and modeling of the background fit. 


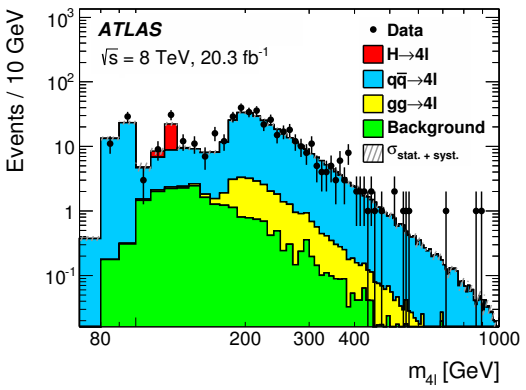

(a)

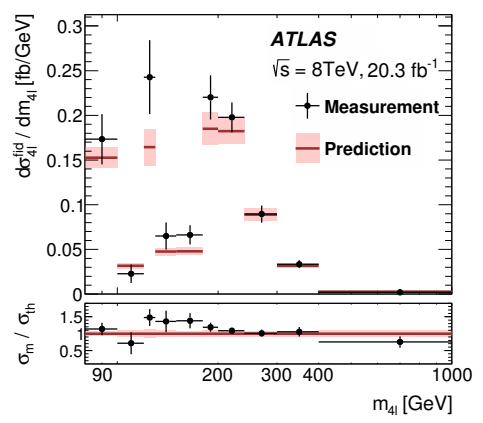

(b)

Figure 4. (a): Data and MC prediction comparison for selected events as a function of the invariant mass $m_{4 l}$ in selected events with four charged leptons. (b): The measured differential cross-section distributions (the black points) of $m_{4 l}$ from events with four charged leptons unfolded into the fiducial volume, and compared to theory predictions (red histogram) [13].

\subsection{Measurement of four lepton production}

There are different ways in $p p$ collisions to produce 4 leptons in the final state. The investigation of this signature is a great way to test the electroweak predictions over a large kinematic range [13]. Events with four charged leptons come from resonant $\mathrm{Z}$ and Higgs decays but also from the nonresonant $\mathrm{ZZ}$ continuum which are produced by $q \bar{q}$ and $g g$ initial states. The selection requires 4 high $p_{\mathrm{T}}$ leptons that form pairs with same flavor but opposite charge in an event. This signature is very clean and the background is very small and arises from events where a lepton is "faked". To get an estimate of this background a data driven technique is used. The resulting invariant mass of the four lepton system can be seen in Figure 4(a) and shows that the different processes contribute in different kinematic regions. Figure 4(b) shows the resulting differential cross section as a function of the invariant mass of the four lepton system in a range from $80 \mathrm{GeV}$ to $1 \mathrm{TeV}$ in comparison with the theory prediction which is overall in good agreement with data. Since the measurement is also sensitive to high $m_{4 l}$ it is possible to estimate the gluon contribution above $180 \mathrm{GeV}$ which is only known at LO QCD precision. In this region a fit to data is performed that takes all non $g g$ contribution and background into account. The cross section taking the integrated number of events from the fit is divided by the LO theory prediction. The result is $\mu_{g g}=2.4 \pm 1.0$ (stat.) \pm 0.5 (syst.) \pm 0.8 (theory) which shows that this measurement can be used to constrain the theory prediction of the $g g$ to four lepton process.

\section{Vector Boson Fusion (VBF) and Vector Boson Scattering (VBS)}

\subsection{Measurement of electroweak production of $Z$ boson in association with two jets}

The production of a $\mathrm{Z}$ boson with two additional jets is mainly dominated by the Drell Yan process where additional jets arise from strong interaction (e.g. gluon radiation) but there is also the possibility to produce a $\mathrm{Z}$ boson + two jets via the electroweak vector boson fusion. Since there is large interference between these two processes it is not possible to completely separate those. The main goal of the analysis is on the one hand to provide the inclusive and differential cross section for $Z j j$ events that provide constraints on the theoretical modeling of QCD-initiated processes and on the other hand to measure the electroweak $Z j j$ production cross section [14]. Therefore the selection of 


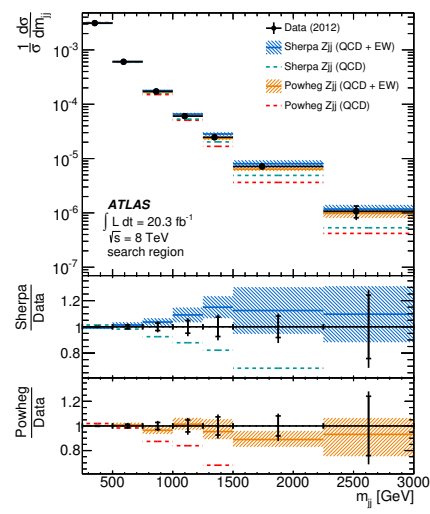

(a)

Figure 5. Unfolded normalized differential cross section distribution as a function of dijet invariant mass of the electro weak production of a $\mathrm{Z}$ boson with two jets [14].

events is divided in different regions that have different sensitivities to the electroweak process. The base line is just the requirement of a pair of high $p_{\mathrm{T}}$ leptons within in the $\mathrm{Z}$ mass range and moderate cuts on the jet $p_{\mathrm{T}}$. Due to the special kinematics of VBF processes the two jets tend to scatter into very forward and backward regions, which leads to large invariant masses of the jet jet system due to large rapidity separation between them. In addition no further hadronic activity is expected between the jets. Thus cuts on $m_{j j}$ and the numbers of additional jets between the forward-backward jets leads to larger sensitivity for electroweak VBF. A result of the differential cross-section in the most sensitive region is shown in Figure 5 where it is visible that a description of the spectrum is not possible with only $Z j j$ QCD processes but predictions by POWHEG and SHERPA are in good agreement when adding the EW processes. The inclusive cross section of electroweak $Z j j$ is found to be $54.7 \pm 4.6(\text { stat. })_{-10.4}^{+9.8}($ syst. $) \pm 1.5$ (lumi. $) \mathrm{fb}$, which is in good agreement with the theory prediction. Therefore limits on anomalous triple gauge couplings were set.

\subsection{Measurement of $W^{ \pm} W^{ \pm} j j$ production}

A key process to check the nature of electroweak symmetry breaking is the scattering of two massive vector bosons (VBS). Therefore an analysis was performed that shows first evidence of VBS contributions in a $W^{ \pm} W^{ \pm} j j$ end state [15]. The required final state consists of two $\mathrm{W}$ bosons and two jets and is dominated by QCD contributions. For VBF the jets tend to have large $p_{\mathrm{T}}$ and a large rapidity gap so that selecting events with high $m_{j j}$ or large rapidity differences of the jets enhances the VBF contribution. In order to suppress background from Drell Yan and $t \bar{t}$ production it is required that the leptons have same charge. The cross section is measured in two different kinematic regions: One more inclusive, containing also QCD contributions, and one with an additional cut on the rapidity difference, $\left|\Delta y_{j j}\right|>2.4$ (VBS region). For the VBS region the significance of the combined signal and background over the background only hypothesis is 3.6 standard deviations. The resulting cross section, measured in the inclusive and the VBS region, can be seen in Figure 6 and is found to be in good agreement with theory prediction within the given uncertainties that are mainly dominated by statistical uncertainties. Therefore limits are set on anomalous quartic gauge couplings. 


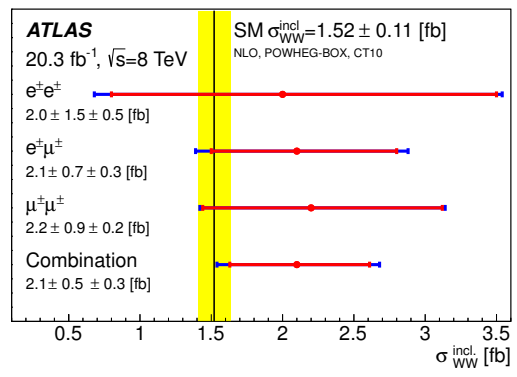

(a)

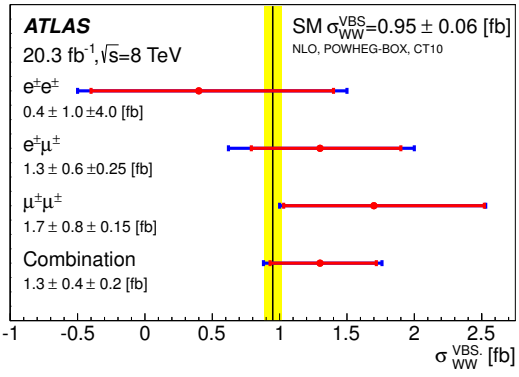

(b)

Figure 6. The measured cross sections of $W^{ \pm} W^{ \pm} j j$ production are shown for the inclusive region (a) and the VBS region (b) compared to the predicted Standard Model cross section [15]. The inner red error band represents the statistical error, and the blue band represents the total error on each measurement. The uncertainties on the cross sections quoted are statistical and systematic respectively.

\subsection{Evidence of $W \gamma \gamma$ production}

Due to the non-Abelian structure of the electroweak sector of the Standard Model also quartic gauge couplings are allowed. Deviations from this SM expectation would be a hint for physics beyond the Standard Model. Therefore an analysis was performed that looks for $W \gamma \gamma$ production in 2012 data of ATLAS with $\sqrt{s}=8 \mathrm{TeV}$ [16]. $W \gamma \gamma$ candidates are formed by requiring a high $p_{\mathrm{T}}$ lepton, large $E_{\mathrm{T}}^{\text {miss }}$ and two isolated photons. Contributions to this event topology can arise from W production with photons emitted in the initial or final state and from triple or quartic gauge coupling processes. The cross section of the process is measured in an inclusive region where no restriction on the recoil system is done and an exclusive region with the restriction that there is no additional jet with $p_{\mathrm{T}}>30 \mathrm{GeV}$ in the event which is more sensitive to quartic gauge couplings. The main background contribution originates from events where jets are mis-identified as leptons, which is estimated using a data driven approach. The invariant mass system of the two photons, shown in Figure 7, shows good agreement between data and expectation when taking the $W \gamma \gamma$ signal contribution into account. The combined significance of signal plus background over background only hypothesis is above $3 \sigma$. The total inclusive cross section is measured to $6.1_{-1.0}^{1.1}($ stat. $) \pm 1.2$ (syst.) \pm 0.2 (lumi.) fb which is in agreement with the theory prediction. Since no significant differences between measurement and expectation are found, limits where set on anomalous quartic gauge couplings.

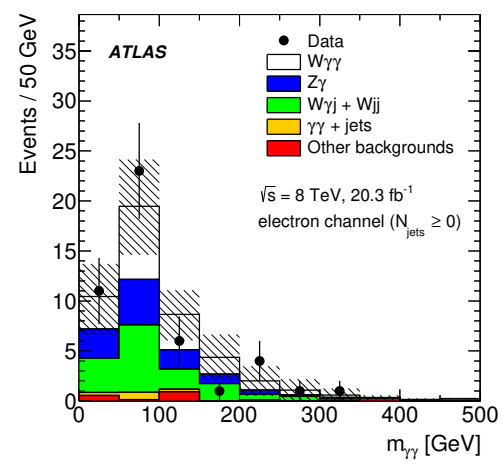

Figure 7. (a): Diphoton invariant mass distribution of selected $W \gamma \gamma$ events in the electron channel. The expected signal based on the SHERPA prediction is shown. The hashed areas show the total systematic and statistical uncertainty on the background estimate [16]. 


\section{Conclusion and Outlook}

Different diboson cross section measurements have been presented. The production cross section of $\mathrm{WZ}$ and WW decaying leptonically and semi-leptonically were compared to theory predictions at $\sqrt{s}=8 \mathrm{TeV}$ and no deviations were found to the Standard Model expectation. Also a differential cross section measurement of $\mathrm{W} \gamma$ and $\mathrm{Z} \gamma$ production using data at $\sqrt{s}=7 \mathrm{TeV}$ is in agreement with theory predictions and could be used for further theoretical constraints. A differential cross section measurement of the invariant mass spectrum of four charged leptons was presented, which can be used as input for theoretical modeling.

A measurement of the $Z j j$ production cross section at $\sqrt{s}=8 \mathrm{TeV}$ was shown, which includes contributions from vector boson fusion processes, and is in good agreement with the Standard Model expectation. An additional measurement looking at processes with $W^{ \pm} W^{ \pm} j j$ in the final state showed the first evidence of electroweak vector boson scattering in this channel. First evidence of electroweak $W \gamma \gamma$ production via quartic gauge coupling was observed which is in good agreement with the Standard Model expectation.

All presented measurements are summarized in Figure 8. It can be seen that the LHC gives a great opportunity to measure electroweak processes over very large range of production cross section. Since there is no deviation to the SM found, limits are set on anomalous triple or quartic gauge couplings. ATLAS data taking restarted in 2015 with $\sqrt{s}=13 \mathrm{TeV}$. The higher center of mass energy results in higher cross section for the electroweak processes and once enough data is taken, there are also new channels where the electroweak sector of the Standard Model can be tested.

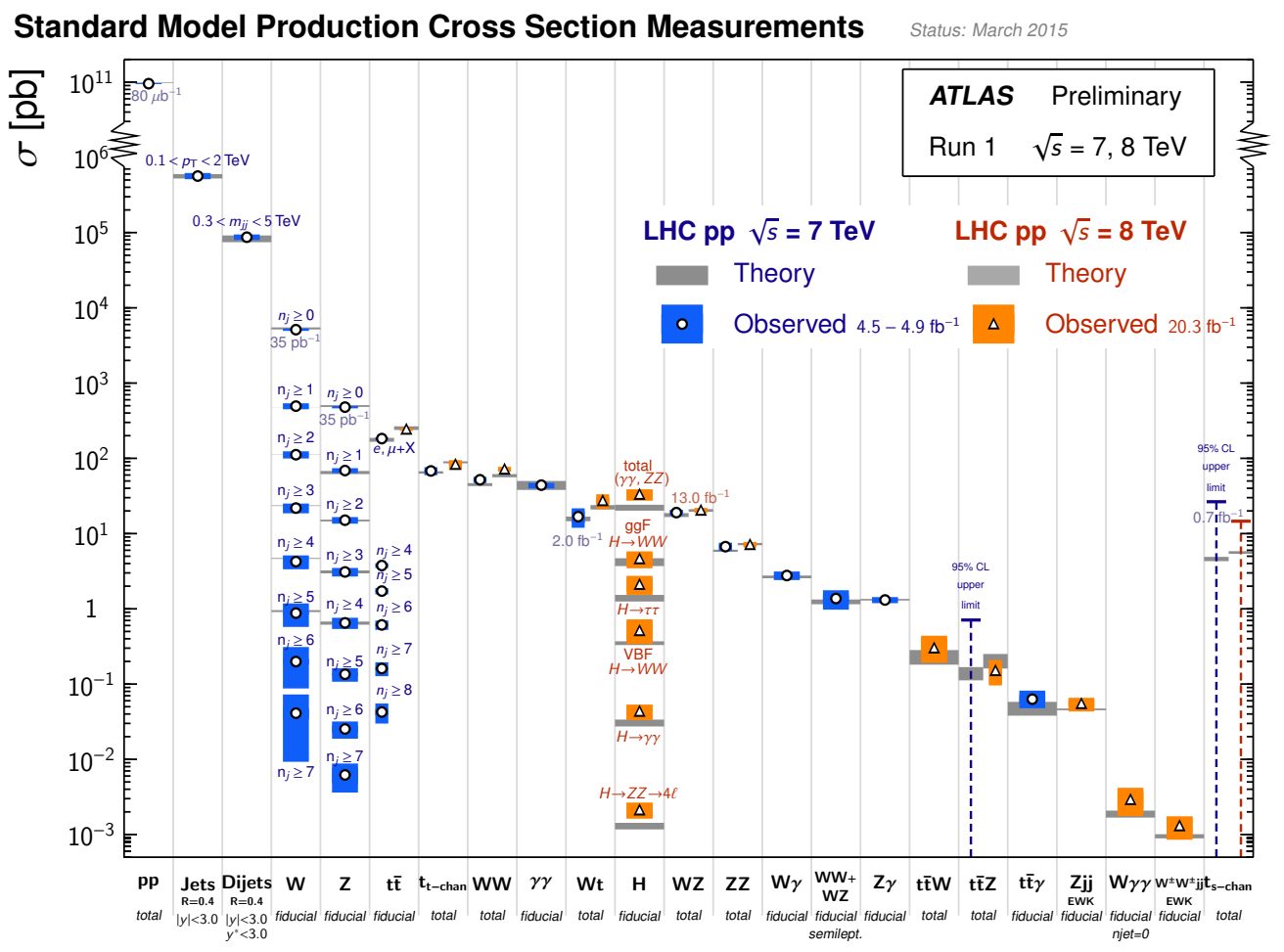

Figure 8. Summary of several Standard Model total and fiducial production cross section measurements, corrected for leptonic branching fractions, compared to the corresponding theoretical expectations. All theoretical expectations were calculated at NLO or higher. [17]. 


\section{References}

[1] ATLAS Collaboration, JINST 3, S08003 (2008)

[2] M. Cacciari, G.P. Salam, G. Soyez, JHEP 04, 063 (2008)

[3] ATLAS Collaboration, Phys. Rev. D 87, 112003 (2013)

[4] T. Gleisberg et al., JHEP 02, 056 (2004)

[5] M.L. Mangano et al., JHEP 07, 001 (2003)

[6] J.M. Campbell, R.K. Ellis, Nucl. Phys. Proc. Suppl. 205-206, 10 (2010)

[7] ATLAS Collaboration, ATLAS-CONF-2013-021, http://cdsweb.cern.ch/record/1525557 (2013)

[8] ATLAS Collaboration, ATLAS-CONF-2014-033, http://cdsweb.cern.ch/record/1728248 (2014)

[9] T. Gehrmann et al., Phys. Rev. Lett. 113, 212001 (2014)

[10] P. Jaiswal, T. Okui, Phys. Rev. D90, 073009 (2014)

[11] ATLAS Collaboration, JHEP 2015, 49 (2015)

[12] S. Frixione, B.R. Webber, JHEP 2002, 029 (2002)

[13] ATLAS Collaboration, arXiv:1509.07844 (2015)

[14] ATLAS Collaboration, JHEP 04, 031 (2014)

[15] ATLAS Collaboration, Phys. Rev. Lett. 113, 141803 (2014)

[16] ATLAS Collaboration, Phys. Rev. Lett. 115, 031802 (2015)

[17] https://atlas.web.cern.ch/Atlas/GROUPS/PHYSICS/CombinedSummaryPlots/SM/ (2015) 